\title{
Magnetotransport Subband Spectroscopy in InAs Nanowires
}

\author{
Florian Vigneau, ${ }^{1}$ Vladimir Prudkovkiy, ${ }^{1}$ Ivan Duchemin, ${ }^{2}$ Walter Escoffier, ${ }^{1}$ Philippe Caroff, ${ }^{3,4}$ Yann-Michel Niquet, ${ }^{2}$ \\ Renaud Leturcq, ${ }^{3}$ Michel Goiran, ${ }^{1}$ and Bertrand Raquet ${ }^{1}$ \\ ${ }^{1}$ Laboratoire National des Champs Magnétiques Intenses, INSA UPS UJF CNRS, UPR 3228, Université de Toulouse, \\ 143 Avenue de Rangueil, 31400 Toulouse, France \\ ${ }^{2}$ L-Sim, SP2M, UMR-E CEA/UJF-Grenoble 1, INAC, 17 Rue des Martyrs, 38054 Grenoble, France \\ ${ }^{3}$ Institute of Electronics Microelectronics and Nanotechnology, CNRS-UMR 8520, ISEN Department, \\ Avenue Poincaré, CS 60069, 59652 Villeneuve d'Ascq Cedex, France \\ ${ }^{4}$ Department of Electronic Materials Engineering, Research School of Physics and Engineering, \\ The Australian National University, Canberra, Australian Capital Territory 0200, Australia
}

(Received 17 October 2013; published 20 February 2014)

\begin{abstract}
We report on magnetotransport measurements in InAs nanowires under a large magnetic field (up to $55 \mathrm{~T}$ ), providing a spectroscopy of the one-dimensional electronic band structure. Large modulations of the conductance mediated by a control of the Fermi energy reveal the Landau fragmentation, carrying the fingerprints of the confined InAs material. Our numerical simulations of the magnetic band structure consistently support the experimental results and reveal key parameters of the electronic confinement.
\end{abstract}

DOI: 10.1103/PhysRevLett.112.076801

PACS numbers: 73.22.-f, 72.15.Gd, 73.63.Nm

Introduction.-Semiconducting nanowires (SC-NW) represent a particular class of nano-objects with a broad range of potential applications in nanoelectronics and optoelectronics [1,2]: their aspect ratio facilitates their processing and combines well with the possibility of band structure tailoring and carrier doping. In particular, small band gap III-V SC-NWs such as InAs present key characteristics, deriving from their wide Bohr radius together with a strong spin-orbit interaction and a large Landé factor. The resulting unusual one-dimensional (1D) electronic band structures constitute the foundation for spin and charge control in future nanodevices [3,4]. Recently, they have been exploited in the field of the quest for Majorana fermions [5].

Tailoring a reduced number of conducting channels in 1D devices remains a challenge. It requires an electronic confinement that comes with a severe drop of mobility [6]. Despite tremendous effort, backscattering on defects is enhanced for the narrowest wires, concealing the 1D electronic properties [6,7]. Only recently, the first signatures of quasi-1D subbands in InAs NWs have been observed as steps in the conductance [8]. However, an exhaustive direct spectroscopy of the confined states in individual NWs remains unachieved.

Here, our strategy consists of playing with InAs NW-based transistors in the open quantum dot regime and under extremely large magnetic field. High magnetic fields are required for a full spin and orbital degeneracy lifting once the magnetic confinement overcomes the electronic one [9]. For a perpendicular magnetic field, associated to a magnetic length $l_{B}=\sqrt{\hbar / e B}$, much smaller than the NW diameter, the 1D electronic band structure evolves into magnetoelectric subbands with a flattening of the dispersion curves, the onset of conducting chiral edge states, and a Zeeman splitting together with an up-shift of the subbands energies accompanying the Landau diamagnetism [10]. These magnetic states modify the conductance in a complex manner, following the depletion of the 1D channels and their degeneracy lifting in the quantum Hall regime [10,11].

In this Letter, we give evidence of the 1D band structure of InAs NWs on the two-probe conductance mediated by the carrier density and a perpendicular magnetic field. Large conductance modulations reveal the magnetic field dependence of the 1D conducting states and the spin and orbital degeneracy. Under large magnetic field and/or at low carrier concentration, a full magnetic depletion of the NW is also achieved, inducing a conductance turning off. Our results are consistently supported by numerical simulations of the magnetic band structure, revealing the key parameters of the electronic confinement in InAs.

InAs NWs with diameters $(D)$ of $30 \pm 5 \mathrm{~nm}$ are synthesized by gold-assisted gas-source molecular beam epitaxy on $\operatorname{InP}(111) \mathrm{B}$ substrates [12] and exhibit a pure wurtzite crystal structure [13]. The NWs are deposited on a degenerately $n$-doped $\mathrm{Si} / \mathrm{SiO}_{2}(225 \mathrm{~nm})$ wafer. $\mathrm{Ti} / \mathrm{Au}$ $(10 / 150 \mathrm{~nm})$ contacts are patterned along the NW by electron beam lithography. The conductance versus backgate voltage is measured from 300 down to $2 \mathrm{~K}$, using the standard lock-in technique in the low bias voltage regime $\left(e V_{b}<k T\right)$ and under controlled atmosphere. The magnetoconductance (MC) in a perpendicular configuration is measured under a $55 \mathrm{~T}$ pulsed magnetic field at a fixed back-gate voltage. We get rid of the unwanted quantum interference effects on the (magneto-)conductance by focusing on measurements above $30 \mathrm{~K}$. Several devices $(>10)$ 
have been fully characterized, ensuring the robustness of our findings. In what follows, we mainly present extensive results for one device defined by a $225 \mathrm{~nm}$ source-drain distance and $D=31 \mathrm{~nm}$, having the hallmarks of the overall samples.

The transfer characteristics $G\left(V_{g}\right)$ are presented in Fig. 1 for selected temperatures (black lines). Clear plateaus of conductance develop below $100 \mathrm{~K}$. They constitute a straightforward signature of successive 1D channels in a (quasi-)ballistic regime. Below $30 \mathrm{~K}$ (not shown here), universal conductance fluctuations conceal the steplike behavior of the conductance. With the use of the Landauer approach, the conductance is expressed by $G=2 e^{2} / h \sum \int-T_{i}(E) \partial f(E) / \partial E d E$, where $f(E)$ is the Fermi-Dirac distribution. The summation includes the 1D modes (i) below the Fermi energy $E_{F}$, and $T_{i}(E)$ refers to their transmission coefficient. The band structure of a $31 \mathrm{~nm}$ diameter defect-free InAs NW is calculated using the $s p^{3} d^{5} s^{*}$ tight-binding model [14]. The dispersion curves are plotted in Fig. 2(a), left. Note that the second subband is twofold degenerated as a consequence of the cylindrical symmetry. The determination of $E_{F}$ for a given $V_{g}$ voltage is deduced from the integrated density of states equal to the number of extra electrons provided by the electrostatic gate $n=C_{g}\left(V_{g}-V_{t}\right) / e$, where $C_{g}$ is the gating efficiency of the InAs device and $V_{t}$ is the threshold voltage. A homogeneous charge carrier distribution is assumed since the accumulation layer in InAs NWs starts to be pronounced for diameters above $40 \mathrm{~nm}$ [7]. The modeling of the conductance with $T_{i}$ and $C_{g}$ as adjustable parameters (red curves, Fig. 1) consistently reproduces the experimental results for all the temperatures. The transmission coefficients we extract per spin channel for the two first subbands are roughly temperature independent and equal to
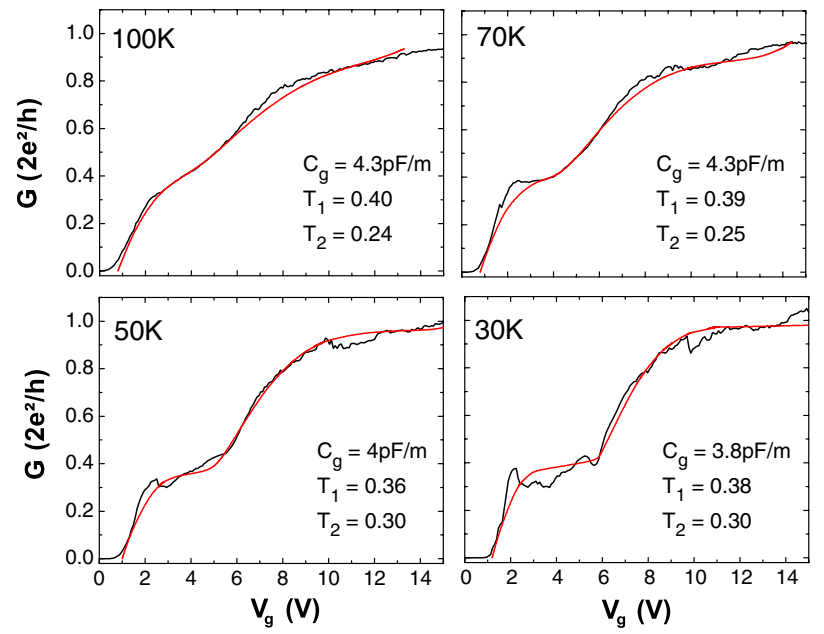

FIG. 1 (color online). Conductance versus $V_{g}$ measured at different temperatures on a $31 \mathrm{~nm}$ diameter InAs NW device (black curves). Red curves are the simulated conductance following the Landauer formula. The electrostatic coupling and the transmission coefficients of the first two subbands are adjustable parameters.
$T_{1,(2)}=0.38(0.28) \pm 0.02$. These values are similar to those already reported on similar InAs NW devices [8]. Assuming independent scattering events between the 1D channels and a negligible contact resistance checked by four-probes measurements (not shown here), we deduce the electronic mean free path $l_{1(2)} \approx 140(90) \mathrm{nm}$ for the first (second) subband, confirming the quasiballistic regime. The modeling of the conductance gives also an estimate of the geometrical capacitance. The knowledge of $C_{g}$ remains a key issue for an accurate determination of the field effect mobility [6]. Here, we deduce an effective coupling of $4.2 \pm 0.2 \mathrm{pF} / \mathrm{m}$, smaller than the simulated one $(13.7 \mathrm{pF} / \mathrm{m})$, taking into account the screening of the metallic electrodes [15] but modeling the nanowire as a metal. Our $G\left(V_{g}\right)$ analysis finally validates the sequence of the calculated conduction subbands minima and provides an accurate correspondence between $E_{F}$ and $V_{g}$.

We now focus on the $\mathrm{MC}$ at high field, which is the main result of our work. In Fig. 3, we plot the measured conductance as a function of the magnetic field applied perpendicular to the NW and for different carrier densities, following the color marks on the $G\left(V_{g}\right)$ plot. Large conductance modulations develop as a function of the magnetic field and depend on the number of 1D channels below $E_{F}$. We schematically define three energy windows exhibiting distinct MC behavior. (i) Between the threshold voltage and the first conductance subband $\left(1 \mathrm{~V} \leq V_{g} \leq 3 \mathrm{~V}\right)$, a monotonic decrease of the conductance is observed with a shift toward high magnetic field when the carrier density is increased. (ii) Below and in the vicinity of the second subband $\left(3.5 \mathrm{~V} \leq V_{g} \leq 6 \mathrm{~V}\right)$, an oscillatory behavior of the conductance develops and consistently shifts to higher field when $E_{F}$ increases. These oscillations go along with an overall increase of the conductance followed by a drop of conductance at high field. (iii) Well inside the second subband $\left(6 \mathrm{~V}<V_{g}\right)$, the oscillatory behavior persists, accompanying an overall
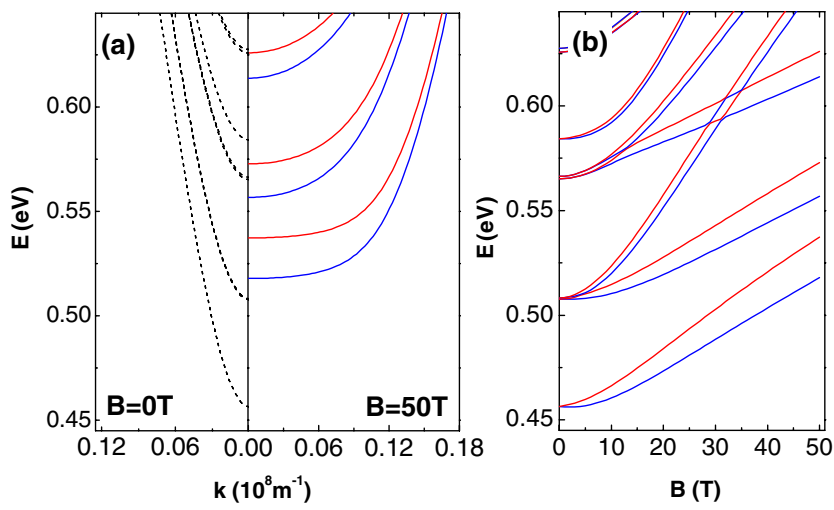

FIG. 2 (color online). (a) Simulated $E(k)$ dispersion curves along the NW axis for a $31 \mathrm{~nm}$ diameter InAs NW, at $0 \mathrm{~T}$ (black dotted lines) and at $50 \mathrm{~T}$ (red and blue lines for up and down spin states, respectively). (b) The corresponding magnetoelectric subbands $E_{i}(B)$ from 0 to $50 \mathrm{~T}$. 


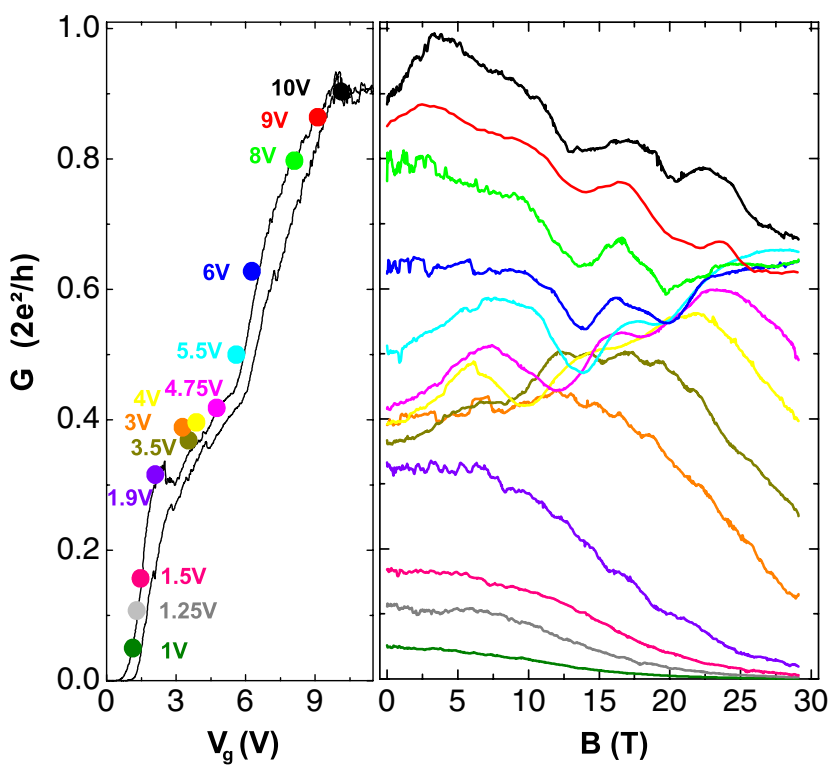

FIG. 3 (color online). Perpendicular MC measured on the $31 \mathrm{~nm}$ InAs NW device at $50 \mathrm{~K}$, for selected back-gate voltages (right panel), following the color marks on the $G\left(V_{g}\right)$ plot (left panel).

decrease of the conductance. Interestingly, several $\mathrm{MC}$ curves, with $5 \mathrm{~V} \leq V_{g} \leq 9 \mathrm{~V}$, merge together above $25 \mathrm{~T}$, suggesting a similar magnetic conducting state.

In the following, we will show that the complex structure of the $\mathrm{MC}$ and, in particular, the oscillating features and the decrease at high field are direct experimental signatures of the crossing of the Fermi level with the magnetoelectric subbands. Figure 3 can then be seen as a fingerprint of the dispersion relation of the confined electrons. For this purpose, we first simulate the magnetic field dependence of the $E_{i}(k)$ dispersion and their energy minima, $E_{i}(B)$ (Fig. 2). Under $50 \mathrm{~T}$, the magnetic length $(3.5 \mathrm{~nm})$ becomes much smaller than the NW radius. The electronic structure fragments into an unusual Landau spectrum, carrying the fingerprints of the confined InAs material. We clearly observe the flattening of the $E(k)$ curves at the $\Gamma$ point, reflecting the degenerate bulk Landau states, and the formation of the dispersive edge states when $k l_{B}{ }^{2}$ approaches the flanks of the wire [Fig. 2(a), right]. The $E_{i}(B)$ curves reveal an overall up-shift driven by the Landau diamagnetism and a strong spin and orbital degeneracy lifting in the presence of a large $|g *|$ factor. We now compare the MC curves with the predicted magnetoelectronic subbands and the calculated field dependence of the Fermi energy $E_{F}(B)$. We assume a constant charge carrier density imposed by $V_{g}$.

Figure 4(a) presents the analysis of the low doped regime (i). On the top panel, we plot the MC curves for $V_{g}$ equal to $1.25,1.9$, and $3 \mathrm{~V}$. The corresponding $E_{F}(B)$ values are superimposed to the magnetic field dependence of the first subband. We clearly observe an increase of $E_{F}$ with a slight pinning when crossing the bottom of the spin degeneracy
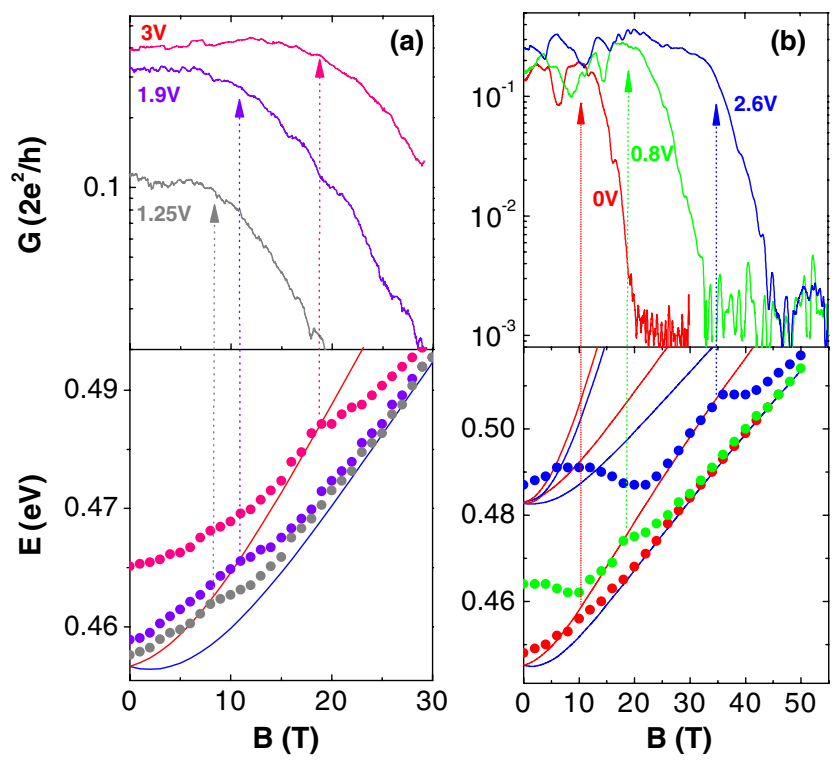

FIG. 4 (color online). (a) Zoom on the MC in the low doping regime measured on the $31 \mathrm{~nm}$ diameter InAs NW device at $50 \mathrm{~K}$ (top panel). Simulated magnetoelectronic subbands and the corresponding $E_{F}(B)$ (bottom panel). The arrows indicate the crossing of $E_{F}$ with the subbands, concomitant with the conductance decrease. (b) MC measurements at $4 \mathrm{~K}$ on a $33 \mathrm{~nm}$ InAs NW device for selected gate voltage, revealing the switch off of the conductance (top panel). The corresponding $E_{F}(B)$ crossing the magnetoelectric subbands demonstrate the magnetic depopulation (bottom panel).

lifted subband. The beginning of the conductance decrease coincides with the loss of the spin-up channel (marked by arrows). At $4 \mathrm{~K}$ [Fig. 4(b), top panel], the drop of conductance, shown on another device with a comparable diameter $(33 \mathrm{~nm})$, is even more sharper. It goes along with the pinning of $E_{F}$ on the lowest level [Fig. 4(b), low panel]. We assign the magnetic quench of the conductance to a complete electronic depletion of the wire. In this regime, the carrier density can not be considered constant anymore. The quantum capacitance starts to be smaller than the geometrical capacitance in series, inducing a strong decrease of the effective coupling and a depletion of the number of free electrons. We numerically checked that the quantum capacitance only plays a role at the bottom of the lowest subband, which means that our simulated $E_{F}(B)$ remains valid until the high-field drop of conductance. We finally emphasize that the magnetic-field-induced scattering by surface defects plays a minor role in the conductance drop since the magnetic quench is also observed for a parallel magnetic field to the NW axis.

In the high doping regime (iii), when $E_{F}$ is well above the second subband edge, the MC exhibits several bumps and steplike decreases [Fig. 5(a), top panel]. Here again, the crossing of $E_{F}(B)$ with the magnetoelectric subbands [Fig. 5(a), lower panel] reveals that all the successive drops of conductance are explained by the subband 

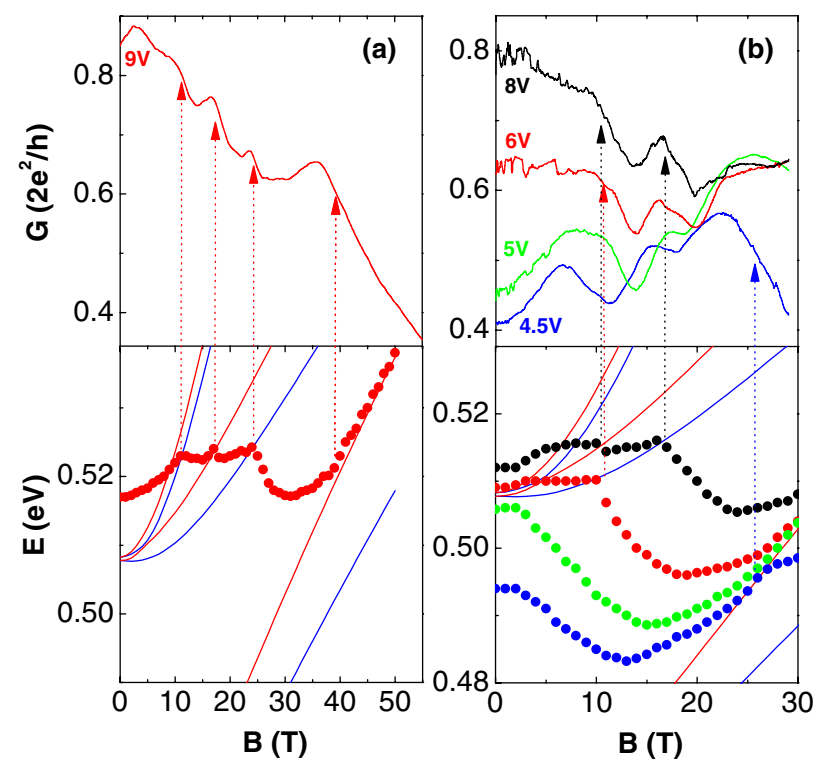

FIG. 5 (color online). (a) Zoom on the MC in the high doping regime $(9 \mathrm{~V})$ measured on the $31 \mathrm{~nm}$ diameter InAs NW device (top panel). The arrows indicate the successive drop of conductance unveiling the loss of conducting channels and the spin and orbital degeneracy lifting of the second subband (bottom panel). (b) Zoom on the intermediate doping regime (top panel). Only few structures of the $G(B)$ curves, marked by arrows, can be explained by the crossing of $E_{F}$ with magnetoelectric subbands (bottom panel).

depopulations. An almost complete degeneracy lifting of the fourfold degenerate second subband is also resolved.

The exact shape of the MC requires a more complex analysis. The Landauer formalism to model the $G(B)$ curves only holds for defect-free NWs and results in a quantized steplike decrease of the conductance, following the magnetic depletion of the subbands. In our case, the successive decreases are not quantized in units of $e^{2} / h$ and they are systematically preceded by a slight increase of the conductance. This can be qualitatively understood in the frame of the Landau fragmentation in presence of disorder. When $E_{F}$ lies between two magnetoelectric sublevels, the onset of chiral channels at the flanks induces a backscattering probability decrease. However, in our case, the subband depopulations occur well before a complete spatial separation of the $k+$ and $k$ - propagative states, preventing any quantized value of the conductance. A quantitative description of the magnetic field dependence of the transmission coefficients would require further developments, out of the scope of this study.

In the intermediate regime (ii), below and in the vicinity of the second subband, the behavior of the MC both confirms the magnetic depopulation and reveals the quantitative limits of our band structure simulations [Fig. 5(b)]. At $8 \mathrm{~V}$, the first and the second drop of conductance, around 10 and $17 \mathrm{~T}$ (marked by the black arrows), corresponds to the loss of the three first conducting channels and the fourth one of the second subband, respectively. At $6 \mathrm{~V}$, the drop of conductance occurring at $10 \mathrm{~T}$ is assigned to the crossing of the lowest sublevel of the second subband with no signature of a degeneracy lifting (marked by red arrow). However, when $E_{F}$ lies within a few meV below the second subband, at 4.5 and $5 \mathrm{~V}$ (blue and green curves, respectively), we do not expect any crossing of $E_{F}(B)$ with magnetoelectric subbands at moderate magnetic field while some conductance modulations are observed. This apparent discrepancy may originate from the limits of the band structure calculation. In particular, the electric field shall split the second subband levels by a few meV. The fractional occupation of those levels lying a few kT (roughly $15 \mathrm{meV}$ ) above $E_{F}$ might reasonably explain the quantum oscillations at the edge of the subband. The band structure in the high doping level (where $E_{F}$ is well above the second subband edge) remains unaffected by this splitting.

In the following, we bring additional evidence of the magneto-fingerprints of the band structure. First, at low field, before the Landau regime (below $5 \mathrm{~T}$ ), we note that the MC successively varies from a flat signal, for $V_{g} \sim 1.5-3$ and 6-8 $V$, to a large gain of conductance, for $V_{g} \sim 4-5.5$ and 9-10 V (Fig. 3). The flat MC signal occurs when $E_{F}$ lies within the van Hove singularities (around $1.5 \pm 0.5$ and $6 \pm 0.5 \mathrm{~V}$ ). The corresponding $k$ vectors are strongly reduced, and the resulting Lorentz force on the electronic trajectory is poorly effective. When $E_{F}$ is located well inside a conducting subband, much larger $k$ vectors are involved. The gain of velocity favors the classical force and the formation of skipping orbits at the flanks, responsible for the positive low-field MC. This picture also explains the overall increase of $G(B)$ for $V_{g} \sim 4-5.5 \mathrm{~V}$. When $E_{F}$ is well above the highest occupied subband, here the first subband, the number of conducting channels remains constant over a wide magnetic field range and the MC is mainly driven by the gradual onset of spatially separated chiral currents, responsible for a gain of conductance. Additionally, we previously mentioned that several MC curves merge together around $25 \mathrm{~T}$ independently of the doping level, for $V_{g}$ varying from 5 to $9 \mathrm{~V}$. Figure 5 reveals that this is supported by a similar magnetic state when only the two lowest subbands carry the current in the high-field regime.

We finally state that the major MC features find natural explanations in the field dependence of the electronic band structure. The evidence of spin and orbital degeneracy lifting also constitutes an efficient tool to confirm the extracted $g^{*}$ factor from the band structure simulation. The first drop of conductance occurring at roughly $B \sim 10 \mathrm{~T}$ for the curves at 6 and $8 \mathrm{~V}$ coincides with two distinct $E_{F}$ $\left(E_{F, 6 \mathrm{~V}} \sim 509 \pm 2 \mathrm{meV}\right.$ and $\left.E_{F, 8 \mathrm{~V}} \sim 515 \pm 2 \mathrm{meV}\right)$ crossing the two sublevels with similar orbital momentum but opposite spin states. We deduce the effective Landé factor for the second subband $\left[\left|g^{*}\right|=\left(E_{F, 8 \mathrm{~V}}-E_{F, 6 \mathrm{~V}}\right) / \mu_{B} B\right]$, of the order of $8.4 \pm 0.4$. Such a value, obtained in the open 
quantum dot regime, is significantly smaller than the InAs bulk value $\left(\left|g^{*}\right| \approx 15\right)$, due to the transverse confinement $[16,17]$.

Conclusion.-By combining clean InAs NWs FET and a large magnetic field environment, we bring new insight into the magnetotransport spectroscopy of the 1D modes in the open quantum dot regime. A direct comparison with simulations reveals the magnetoelectric subbands and their major impacts on the conductance, from which key parameters such as the $g^{*}$ factor can be deduced. This opens news routes toward a magnetic tailoring of the $1 \mathrm{D}$ band structure in SC-NWs.

Part of this work is supported by EuroMagNET, Contract No. 228043, and the ANR Project No. ANR-11-JS04-00201. The authors thank Xavier Wallart for helpful discussions.

[1] P. Yang, R. Yan, and M. Fardy, Nano Lett. 10, 1529 (2010); N. S. Ramgir, Y. Yang, and M. Zacharias, Small 6, 1705 (2010); Y. Li, F. Qian, J. Xiang, and Ch. M. Lieber, Mater. Today 9, 18 (2006).

[2] S. A. Dayeh, Semicond. Sci. Technol. 25, 024004 (2010).

[3] A. Bringer and Th. Schäpers, Phys. Rev. B 83, 115305 (2011).

[4] P. Streda and P. Seba, Phys. Rev. Lett. 90, 256601 (2003).

[5] V. Mourik, K. Zuo, S. M. Frolov, S. R. Plissard, E. P. A. M. Bakkers, and L.P. Kouwenhoven, Science 336, 1003 (2012); M. T. Deng, C. L. Yu, G. Y. Huang, M. Larsson, P. Caroff, and H. Q. Xu, Nano Lett. 12, 6414 (2012); A. Das, Y. Ronen, Y. Most, Y. Oreg, M. Heiblum, and H. Shtrikman, Nat. Phys. 8, 887 (2012).
[6] A. C. Ford, J. C. Ho, Y.-L. Chueh, Y.-C. Tseng, Z. Fan, J. Guo, J. Bokor, and A. Javey, Nano Lett. 9, 360 (2009); N. Gupta, Y. Song, G. W. Holloway, U. Sinha, C. M. Haapamaki, R. R. LaPierre, and J. Baugh, Nanotechnology 24, 225202 (2013).

[7] S. A. Dayeh, C. Soci, P. K. L. Yu, E. T. Yu, and D. Wang, J. Vac. Sci. Technol. B 25, 1432 (2007); S. A. Dayeh, E. T. Yu, and D. Wang, Small 5, 77 (2009).

[8] A. C. Ford, S. B. Kumar, R. Kapadia, J. Guo, and A. Javey, Nano Lett. 12, 1340 (2012); S. Chuang, Q. Gao, R. Kapadia, A. C. Ford, J. Guo, and A. Javey, Nano Lett. 13, 555 (2013).

[9] S. Nanot, R. Avriller, W. Escoffier, J.-M. Broto, S. Roche, and B. Raquet, Phys. Rev. Lett. 103, 256801 (2009).

[10] E. N. Bogachek, A. G. Scherbakov, and U. Landman, Phys. Rev. B 53, R13246 (1996); J. Knobbe and Th. Schapers, Phys. Rev. B 71, 035311 (2005); Y. Tserkovnyak and B. I. Halperin, Phys. Rev. B 74, 245327 (2006).

[11] M. Royo, A. Bertoni, and G. Goldoni, Phys. Rev. B 87, 115316 (2013).

[12] C. Rolland, P. Caroff, C. Coinon, X. Wallart, and R. Leturcq, Appl. Phys. Lett. 102, 223105 (2013).

[13] T. Xu, K. A Dick, S. Plissard, T. H. Nguyen, Y. Makoudi, M. Berthe, J.-P. Nys, X. Wallart, B. Grandidier, and P. Caroff, Nanotechnology 23, 095702 (2012).

[14] J. M. Jancu, R. Scholz, F. Beltram, and F. Bassani, Phys. Rev. B 57, 6493 (1998); Y. M. Niquet, A. Lherbier, N. H. Quang, M. V. Fernandez-Serra, X. Blase, and C. Delerue, Phys. Rev. B 73, 165319 (2006).

[15] The capacitance of the device including the contacts was calculated by a 3D electrostatic simulation using COMSOL MULTIPHYSICS.

[16] A. A. Kiselev, E. L. Ivchenko, and U. Rössler, Phys. Rev. B 58, 16353 (1998).

[17] S. Csonka, L. Hofstetter, F. Freitag, S. Oberholzer, and C. Schonenberger, Nano Lett. 8, 3932 (2008). 